DOE/ER/54687-3

UW-CPTC 05-6

\title{
Numerical Studies of Magnetohydrodynamic Activity Resulting from Inductive Transients
}

\author{
Final Report \\ August 15, 2002 - August 14, 2005 \\ C. R. Sovinec \\ University of Wisconsin-Madison \\ Madison, WI 53706
}

August 2005

THE U.S. DEPARTMENT OF ENERGY

AWARD NO. DE-FG02-02ER54687

\begin{abstract}
NOTICE
This report was prepared as an account of work sponsored by the United States Government. Neither the United States nor the United States Department of Energy, nor any of their employees, nor any of their contractors, subcontractors, or their employees, makes any warranty, express or implied, or assumes any legal liability or responsibility for the accuracy, completeness, or usefulness of any information, apparatus, product or process disclosed or represents that its use would not infringe privately-owned rights.
\end{abstract}




\title{
Numerical Studies of Magnetohydrodynamic Activity Resulting from Inductive Transients
} U.S. Department of Energy, Office of Science, Contract DE-FG02-02ER54687

Final Report

\author{
Principal Investigator: Carl Sovinec (sovinec@engr.wisc.edu) \\ Department of Engineering Physics, University of Wisconsin-Madison \\ 1500 Engineering Drive, 519 ERB \\ Madison, WI 53706-1609
}

\section{Executive Summary}

This report describes results from numerical studies of transients in magnetically confined plasmas. The work has been performed by University of Wisconsin graduate students James Reynolds and Giovanni Cone and by the Principal Investigator through support from contract DE-FG02-02ER54687, a Junior Faculty in Plasma Science award from the DOE Office of Science. Results from the computations have added significantly to our knowledge of magnetized plasma relaxation in the reversed-field pinch (RFP) and spheromak. In particular, they have distinguished relaxation activity expected in sustained configurations from transient effects that can persist over a significant fraction of the plasma discharge. We have also developed the numerical capability for studying electrostatic current injection in the spherical torus (ST). These configurations are being investigated as plasma confinement schemes in the international effort to achieve controlled thermonuclear fusion for environmentally benign energy production. Our numerical computations have been performed with the NIMROD code (http://nimrodteam.org) using local computing resources and massively parallel computing hardware at the National Energy Research Scientific Computing Center. Direct comparisons of simulation results for the spheromak with laboratory measurements verify the effectiveness of our numerical approach. The comparisons have been published in refereed journal articles by this group and by collaborators at Lawrence Livermore National Laboratory (see Section 4). In addition to the technical products, this grant has supported the graduate education of the two participating students for three years.

\section{Objectives and Accomplishments}

The proposed objectives and related accomplishments are as follows.

\subsection{Pulsed Parallel Current Drive in RFPs}

1) How does the current profile evolve when the electric field pulses are applied? What are the roles of pinching, resistivity profile change, and nonlinear dynamo drive for the current during the transient?

Our computations have investigated pinching and nonlinear dynamo changes in detail. They show that the electric field penetration is initially diffusive, since it is applied parallel the edge magnetic field. However, the shear in the magnetic field prevents significant diffusive penetration, and pinching is significant over most of the plasma volume. The dynamo activity is quickly suppressed, and the reduced fluctuation activity leads to nonlocal changes in the electric field profile during the transient.

2) How does the pressure profile change as dynamo activity is reduced? Do inductive effects such as pinching have significance, or is the improved confinement solely responsible for the evolution? 
Does the pressure gradient have any bearing on the core-resonant $m=1$ modes during the improved operation?

Computations performed early in the study include finite pressure, and they demonstrate improvements in the energy confinement time after application of the electric field pulse. There was no indication of enhanced $m=1$ drive from the changing pressure gradient during the transient, but more work is needed in this area.

3) What is the drive and role of the $m=0$ fluctuations? Do the simulations provide any theoretical basis for operational limits that have been correlated with the excitation of these fluctuations?

Our computations have not shown isolated $m=0$ fluctuations during the transient, unlike the experiment. It is possible that the level of viscous damping used for nonlinear numerical stability at computationally practicable resolution has prevented isolated $m=0$ fluctuations, and this needs to be reconsidered as improvements in algorithms and hardware allow greater resolution.

\subsection{Spheromak Transients}

1) Do inductive effects at finite- $\beta$ influence the MHD fluctuations (and the $n=1$ mode in particular) that are observed in SSPX?

This study has been very successful in demonstrating important inductive effects at finite plasma- $\beta$ (i.e. plasma pressure). Simulations of specific SSPX discharges with realistic current injection waveforms show that relaxation occurs during the initial formation pulse and that the second smaller current pulse effectively delays the onset of harmful resonant MHD activity. However, the second pulse does not sustain current in the closed-flux region, so inductive effects (and temperaturedependent transport effects) are very important.

2) Are the magnetic field configurations produced by the magnetofluid simulations consistent with the electron temperature and energy confinement observed in spheromak experiments?

Detailed comparisons have been made between our magnetofluid simulations of specific SSPX discharges and laboratory observations. The simulations show all of the major features of the experiment (a significant accomplishment for comprehensive plasma simulations where there are no fitting parameters), and reasonable quantitative agreement on the global magnetic evolution including fluctuation-induced flux amplification, the evolution of the electron temperature profile, and magnetic fluctuation levels.

3) Is it possible to optimize pulsed spheromak operation, such that the confinement achieved during decay makes the cycle-average acceptable for a reactor concept?

Our optimization study has not advanced to the point of being able to predict cycle-averaged confinement, but we (including collaborators at LLNL) have shown that different pulsing schemes have a strong influence on the volume and duration of closed-flux regions. 


\subsection{Spherical Torus Startup}

1) Is the MHD activity driven by electrostatic helicity injection similar to earlier predictions in simplified geometry, and what magnetic configurations are produced at small aspect ratio?

Our electrostatic injection study for ST startup has not yet progressed to the degree of the spheromak study. We have made progress in simulating current filament injection from a localized source into a small-aspect ratio geometry, but these simulations require considerably more numerical resolution than toroidally symmetric injection.

2) What happens to the MHD activity during the transition to inductive drive? Can the transition be controlled or optimized to minimize the consumption of volt-seconds?

Our inductive drive study included realistic geometry, vacuum poloidal fields, and time-dependent vertical field. While we have modeled inductive ST startup, the results are very sensitive to transport coefficients. This issue needs to be revisited with temperature-dependent transport coefficients developed for the spheromak modeling and then combined with electrostatic drive.

3) What determines the nonlinear evolution of the internally resonant MHD activity in Pegasus during start-up, and why does it degrade the discharge? Will MHD activity be significant with compressional heating and current drive?

The sensitivity to fixed transport coefficients used in the earlier modeling did not warrant threedimensional computations. This topic also needs to be revisited with the more realistic temperaturedependent transport coefficients.

\section{Summary}

The basic characteristic times of all magnetically confined plasmas are spread over many orders of magnitude. Considering macroscopic activity alone, Alfvénic propagation can be $10^{4}-10^{5}$ times faster than global resistive diffusion in the smallest experiments. With respect to the development of ideal MHD instabilities (except near a threshold), the profile of the equilibrium is therefore essentially fixed. However, there are important instances where equilibria and macroscopic activity evolve on similar timescales and have important interaction. In general, magnetic relaxation is a process whereby non-ideal macroscopic activity changes the topology of the confining magnetic field and the large-scale distribution of charge current density. The underlying nonlinear evolution of non-ideal MHD occurs over time-scales that are closer to global resistive diffusion than to Alfvénic propagation, so diffusion over fractions of the device scale can be significant, especially for smaller experiments and during start-up. The physical phenomena that participate in the evolution may be familiar (magnetic tearing, relaxation, diffusion) while nonlinear interactions among them lead to unique behavior for specific modes of operation in each system. These interactions can be particularly complicated during externally imposed transients, because the electric field profile is time-dependent and possibly very different from typical sustained conditions. In this study, we have applied numerical computation to better understand three experiments where there is strong interaction between global transients and MHD activity. In Section 3.1, we describe our 
findings on pulsed parallel current drive (PPCD) in the RFP. Section 3.2 summarizes results on spheromak transients, and Section 3.3 presents progress on simulating spherical torus start-up and current injection. Section 3.4 provides an appendix on the numerical modeling.

\subsection{Pulsed poloidal current drive in reversed-field pinches}

The RFP configuration with pulsed parallel current drive (PPCD) is an experiment where there is important synergy between induced transients and nonlinear MHD. In the Madison Symmetric Torus (MST, http://plasma.physics.wisc.edu/mst/html/mst.htm), toroidal magnetic flux is removed during discharges through a series of voltage pulses applied over 5-10 ms to the toroidal gap in the conducting shell. In recent experiments, the loop voltage is simultaneously reversed as the toroidal component of edge magnetic field becomes more negative [1]. Standard discharges without the pulsing exhibit episodic relaxation cycles (RFP sawteeth) every 2-10 ms, so the PPCD transient and MHD activity tend to occur over similar time-scales. The intent of the pulsing is to induce parallel current in the edge of the plasma to reduce the parallel current gradient and hence the drive of magnetic fluctuations that affect confinement [2]. The approach has been very successful in MST, where the amplitude of core-resonant modes decreases and energy confinement increases. Some discharges exhibit residual $m=0$ fluctuations (where $m$ is the poloidal Fourier index) that are resonant near the plasma edge, but in contrast to normal operating conditions, it seems unlikely to result from nonlinear coupling of the remaining core-resonant fluctuations. Although core electron temperatures increase relative to standard RFP discharges when the $m=0$ activity occurs, peak temperatures are significantly higher when the fluctuations are absent [1]. When all MHD activity is suppressed, a dynamo-free condition is achieved [3], and confinement reaches record levels for the RFP.

The focus of our numerical study of PPCD [4] has been to understand how the magnetic fluctuation level in MST responds to the transient—applied as an edge electric field—on resistive tearing time-scales [5] when the global diffusion time is on the order of a second. We start from simulations of standard RFP conditions in a periodic cylinder with plasma current sustained by an applied axial electric field. The evolution displays familiar characteristics of large core-resonant $m=1$ tearing modes interacting through $m=0$ fluctuations [6]. Relaxation results from correlated fluctuations of flow velocity and magnetic field that produce the dynamo electric field $\mathbf{E}_{f}=-\langle\tilde{\mathbf{v}} \times \tilde{\mathbf{b}}\rangle$ which suppresses parallel current density in the plasma core and drives it near the edge [7]. The simplest PPCD-like effect is modeled by applying a purely poloidal electric field at an arbitrary time after saturation in a standard RFP simulation. The modeled electric-field pulse is scaled to change the same fraction of axial (toroidal) flux over the same number of tearing times as PPCD transients in MST. [The Lundquist number (S) in the simulations is much smaller than in MST, particularly in the simulations described here where the S-value is 2000.] As shown in Fig. 1, there is an immediate drop in the energy of the $n=6$ fluctuation, which is dominant at the time the poloidal pulse is applied in an $R / a=3$ NIMROD [http://nimrodteam.org] simulation that includes the $0 \leq n \leq 42$ toroidal Fourier components. The initial evolution $\left(0.201 \leq t / \tau_{r} \leq 0.207\right)$ of the normalized parallel current density $\left(J_{\|} / B\right)$, also shown in Fig.1, is very slight and most evident near the wall.

Among the possible stabilization mechanisms for the core-resonant mode, we immediately rule-out the kind of direct stabilization achieved with feedback systems, since the applied electric field pulse is uniform over the problem domain. This leaves two possibilities: either the nonlinear coupling among fluctuations changes to reduce the amplitude of the core-resonant mode, or the slight change in the profile 
is enough to change the linear drive. The first of the two is plausible for modes with either large $n$-values or $m=0$ (not shown) that are linearly stable and driven nonlinearly. However, the $n=6-8$ modes typically supply the nonlinear cascading channels [6]. To verify that the profile change is then the stabilization mechanism, we have computed the evolution of the rate of energy transferred from the equilibrium to the core modes [7], plus dissipation,

$$
-\operatorname{Re}\left\{\int\left[\langle\mathbf{J}\rangle \cdot\left\langle\tilde{\mathbf{v}}_{n}^{*} \times \tilde{\mathbf{b}}_{n}\right\rangle+\langle\mathbf{V}\rangle \cdot\left\langle\tilde{\mathbf{j}}_{n}^{*} \times \tilde{\mathbf{b}}_{n}\right\rangle+\eta \tilde{\mathbf{j}}_{n}^{*} \cdot \tilde{\mathbf{j}}_{n}+\rho v\left(\nabla \tilde{\mathbf{v}}_{n}^{*}\right)^{T}: \nabla \tilde{\mathbf{v}}_{n}\right] d V o l\right\},
$$

where $\eta$ is the electrical resistivity, $v$ is the kinematic viscosity, and $\rho$ is the mass density. This power drops sharply with the application of the PPCD pulse, providing an explanation for the initial drop in the fluctuation energy shown in Fig. 1 and supporting the idea of a prompt reduction of the linear drive despite the weak change in parallel current density.

To verify that the profile evolution has a stabilizing effect on the dominant mode, we evaluate the tearing-mode stability parameter $\left(\Delta^{\prime}\right)$ for zero-beta conditions [8]. The equilibria are taken from the axially and poloidally symmetric part of the magnetic field in the 3D simulation at different points in the PPCD transient and are analyzed with computations similar to those described in Ref. [9]. In Fig. 2, we show the two linearly independent solutions to the outer-region equation (a form of Newcomb's equation) that are computed as solutions to initial value ODEs in the radial coordinate from $r=0$ and from the resonant surface $\left(r_{s}\right)$. The solutions can be combined to construct the eigenfunction that satisfies homogeneous Dirichlet boundary conditions at $r=0$ and $r=a$. The stability parameter is then $\Delta^{\prime}=-\psi_{S}^{\prime}\left(r_{S}\right) \psi_{C}(a) / \psi_{S}(a)$, where $\psi_{s}$ is the small solution on the positive side of the resonant surface, and $\psi_{c}$ is the continuous (large) solution. The deviations from the start of the pulse ( $t=0.201 \tau_{r}$ ) to $t=0.207 \tau_{r}$ for both the small and continuous (initial value) solutions reduce $\Delta^{\prime}$, making the configuration less unstable to this mode. It is worth noting that the deviation between the solutions for the two equilibria occurs more than halfway into the plasma, not far from the resonant surface, $r_{s}=0.27 a$.

While linear stability analysis is insufficient to describe the nonlinear evolution from the saturated RFP state, it provides information on us how the stirring mechanism for magnetic fluctuations is affected by the transient. The Newcomb equation analysis shows that the important influence of the electric pulse is deep in the plasma core-not edge current drive. Since a large part of the saturation of the coreresonant RFP modes comes from relaxation of the current profile [7], the profile is already close to marginal linear stability before the transient is induced. Slight changes to the current profile near the surface of resonance therefore have a significant effect, and in an MHD system, information can be communicated at an Alfvénic rate if the pulse excites any perpendicular forces [effects of which are also reported in Ref. 10]. 

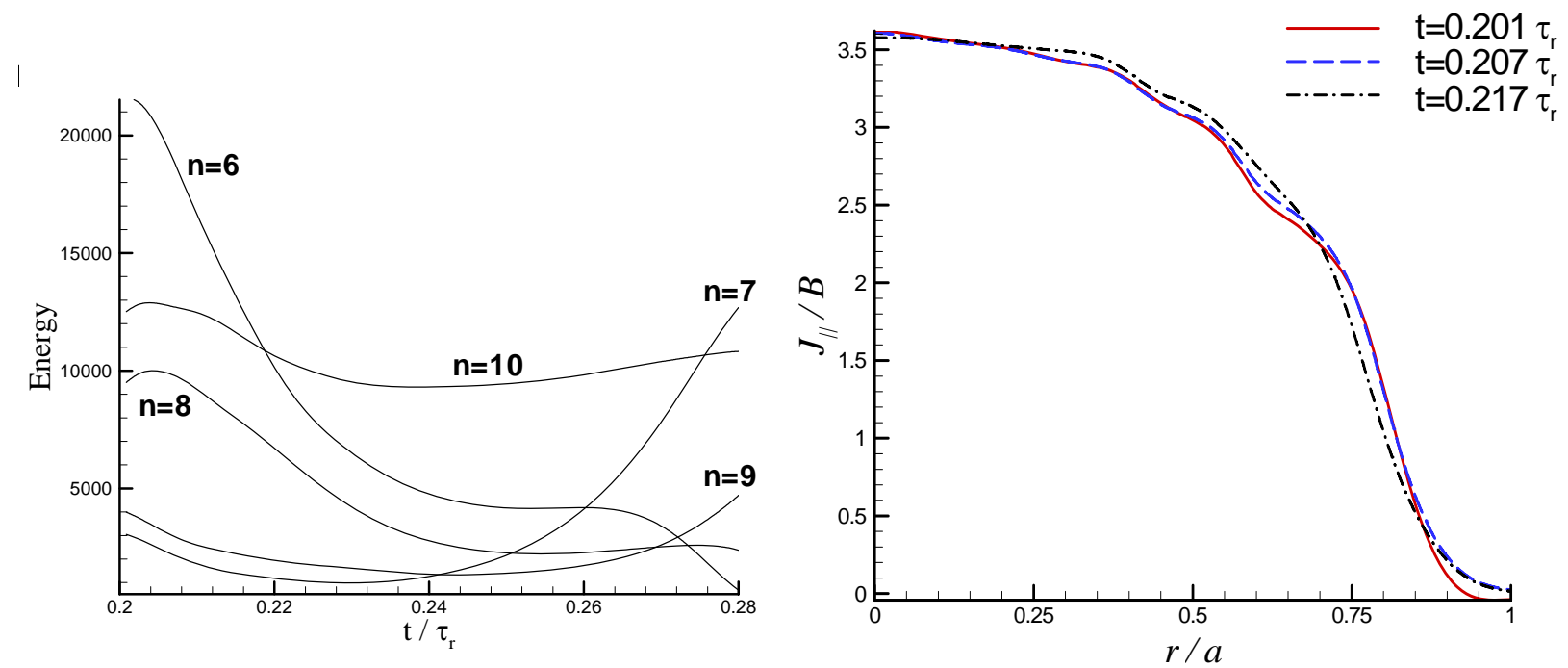

Figure 1. Evolution of magnetic fluctuation energies, labeled by toroidal Fourier component index (left) during the application of a poloidal electric field pulse, and normalized parallel current profiles taken from the initial stabilization phase (right). Times are in resistive diffusion times.
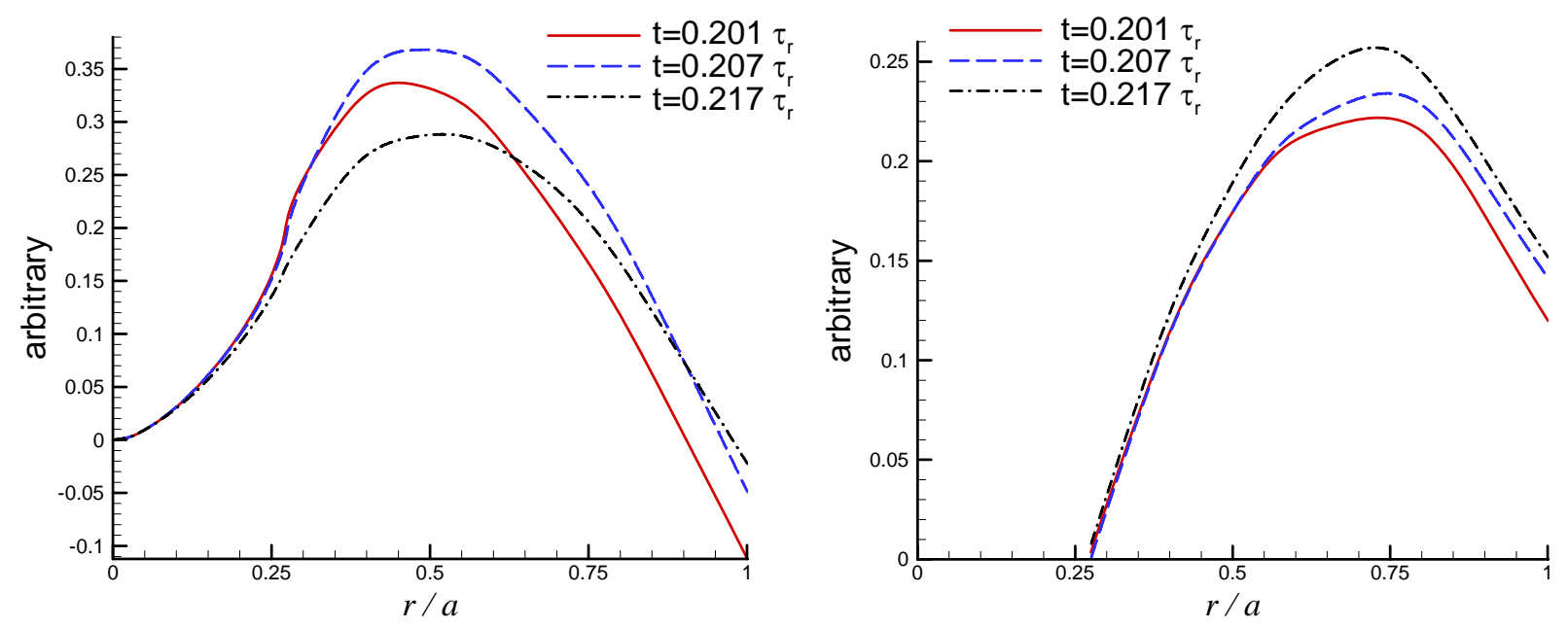

Figure 2. Solutions to linear initial-value ODE problems for the outer regions of the $n=6$ resistive tearing mode for equilibria taken from the 3D nonlinear simulation at the times indicated. The solutions satisfying regularity conditions at $r=0$ with continuous slope at the rational surface are shown on the left, and small solutions with unit slope at the rational surface are shown on the right. 


\subsection{Spheromak evolution}

A transiently enhanced condition is also achieved in the SSPX spheromak (http://www.llnl.gov/str/Hill.html). Like most recent spheromak experiments, SSPX is driven electrostatically from a magnetized plasma gun. Standard operation applies a large formation pulse to expel poloidal and toroidal magnetic flux from the gun and into the large flux conserver, where magnetic relaxation produces the spheromak configuration. The formation pulse lasts approximately $250 \mu$ s, and it is followed by a brief decay period and then a sustainment pulse that is typically at half the injected current of the formation pulse. Like all other electrostatically driven spheromaks [11-13, for example], there are large $n=1$ magnetic fluctuations ( $n$ is the toroidal Fourier index) during formation, due to an MHD kink instability of the pinched column that carries current from one electrode to the other. Fluctuations decrease during the partial decay, as expected from previous results on decaying spheromaks $[11,14]$. However, applying the sustainment pulse leads to a relatively quiescent state at the same time that the decay rate of the configuration decreases [15]. Magnetohydrodynamic activity with $n$-values greater than unity occurs later in the discharge, and specific modes have been correlated to resonant conditions in the evolving q-profile [16], which is computed with CORSICA equilibrium fits to experimental data [17].

Early simulations of spheromak formation and sustainment using the simple 0-beta resistive MHD model demonstrate flux conversion through the $n=1$ kink mode of the electrode-driven current column [18]. In sustained conditions, magnetic field-lines exhibit chaotic scattering [19] across the apparent separatrix of the equilibrium (toroidally averaged) poloidal flux. These simulations also show that closed flux surfaces form when the applied potential is removed [18], due to fluctuations decaying quickly relative to the large-scale fields generated by relaxation. In the sustained open-field configurations, electron temperatures resulting from Ohmic heating with classical parallel transport are limited to tens of electron-Volts [20,21]. Thus, the early simulation results offer an explanation for the high temperatures achieved during the decay phase of some experiments [11,14] but appeared to be inconsistent with the SSPX results where temperatures exceeding $100 \mathrm{eV}$ (and greater than $300 \mathrm{eV}$ more recently) are achieved upon application of a second current drive pulse that follows a brief period of decay [15]. Noting that the second current pulse in SSPX does not keep the magnetic energy content fixed, we developed capabilities for more detailed simulations of SSPX [22] including a realistic geometry, temperature-dependent anisotropic thermal conduction, and a current injection waveform that mimics the formation/sustainment pulsing in SSPX. Temperature evolution is needed for evaluating the temperature dependence of electrical resistivity, and it permits direct comparisons with experimental measurements related to confinement. The heat flux modeling is appropriate for collisional plasmas [23] and relies upon the highorder accuracy of the NIMROD spatial representation [24]. Although limited in its range of validity, the collisional model is appropriate for the lower temperatures (up to $\sim 35 \mathrm{eV}$ ) encountered in regions where the field-lines remain open and globally during the critical transition from open to closed topology [22].

The recent simulations of SSPX represent integrated modeling of nonlinear MHD and energy transport and are applied to specific SSPX discharges starting just after breakdown. The initial magnetic field is just the externally created vacuum bias flux, and the flux amplification and current profile relaxation processes are produced self-consistently (Fig. 3). Besides the effects that were observed in the earlier generic simulations, the recent computations reproduce essentially all of the important characteristics of the corresponding SSPX experiments [15] including: 1) kV voltage spikes during formation, 2) a quiescent phase with magnetic fluctuations at the probe location dropping below 1\%, 3) 
elevated temperatures in a toroidally localized zone during the quiescent phase, and 4) a weak decay of toroidal current and magnetic energy through most of the sustainment pulse [22]. The toroidal rotation of magnetic fluctuations is much smaller in the simulation results than in the experiment, but this appears to be a secondary effect [25]. For a comprehensive model of an entire discharge that is free of any fitting of parameters, the MHD simulations show a remarkable level of quantitative agreement with the laboratory observations.

Having established the relevance of the computational results, we examine the simulated discharge in greater detail to better understand the behavior observed in SSPX. In particular, we compare the dynamo power density [7], $-\langle\tilde{\mathbf{v}} \times \tilde{\mathbf{b}}\rangle \cdot\langle\mathbf{J}\rangle$, during the formation pulse (400 kA) and during the sustainment pulse (200 kA). From Fig. 4, we see that the interaction between fluctuations and the equilibrium current density profile differs by orders of magnitude, despite the fact that the injected current only differs by a factor of two. Near the magnetic axis of the equilibrium $(R \cong 0.35 \mathrm{~m})$, the magnitude of the dynamo power density during formation exceeds resistive loss, $\eta\langle\mathbf{J}\rangle^{2}$, by more than an order of magnitude, so the net toroidal electric field builds equilibrium poloidal flux as part of the relaxation process. However, during the sustainment pulse, the resistive loss exceeds the dynamo power density by more than an order of magnitude; hence the configuration is not sustained. At the reduced resistivity associated with the elevated temperature, the decay time significantly exceeds the pulse length, so decay occurs at the weak rate observed in both experiment and simulation. This nontrivial interaction between the transient, fluctuation-induced relaxation, and energy confinement/temperature-dependent resistivity was only made clear with the detailed information provided by the numerical simulations.

While the second current pulse in SSPX does not prevent decay of the profile, it has a strong role in the evolution of MHD modes that are resonant within the region of amplified poloidal flux (indicated by dashed contours in Fig. 3). The simulation results show that the second pulse prevents the minimum of the non-monotonic $q$-profile from dropping below $1 / 2$. This keeps the $m=1, n=2$ mode and its harmonics from destroying closed flux surfaces that lie inside the separatrix, preserving a topologically distinct confinement region until late in the discharge [22]. The importance of resonant fluctuations and their correlation with SSPX confinement has been noted in the experiment [16], but the simulations have clarified the role of the second current pulse and the fact that the resonant modes are not part of a dynamo process. 
(a)

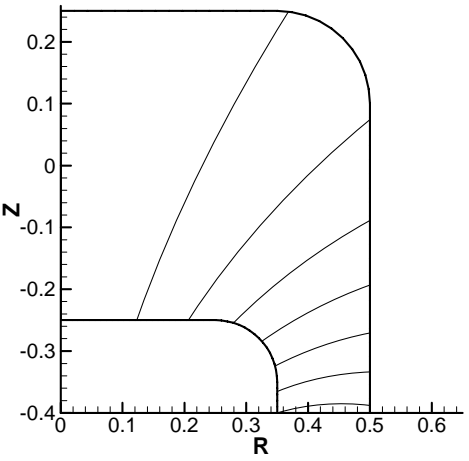

(c)

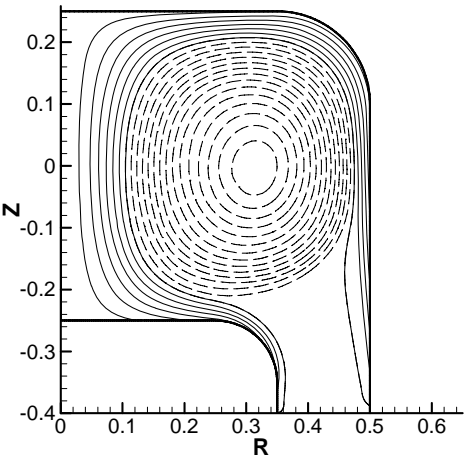

(b)

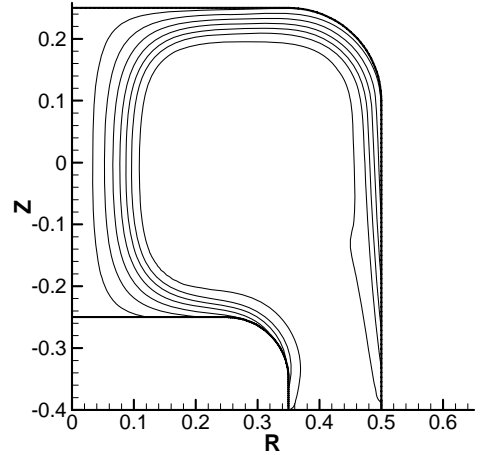

(d)

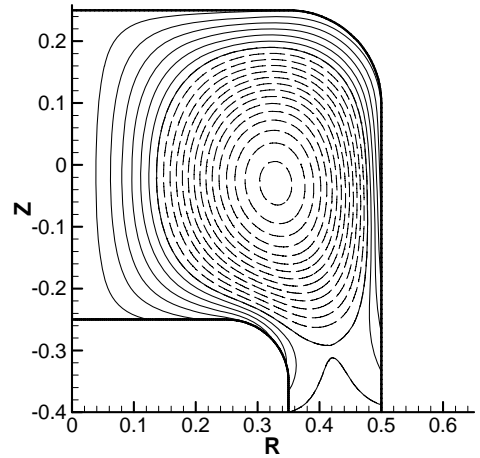

Figure 3. Evolution of the (toroidal average) poloidal flux function from NIMROD simulations of SSPX discharges 4620-4644. The frames show a) the initial vacuum distribution, b) prior to relaxation, c) after relaxation, and d) during application of the sustainment bank. Dashed contours show poloidal flux generated by the relaxation process.

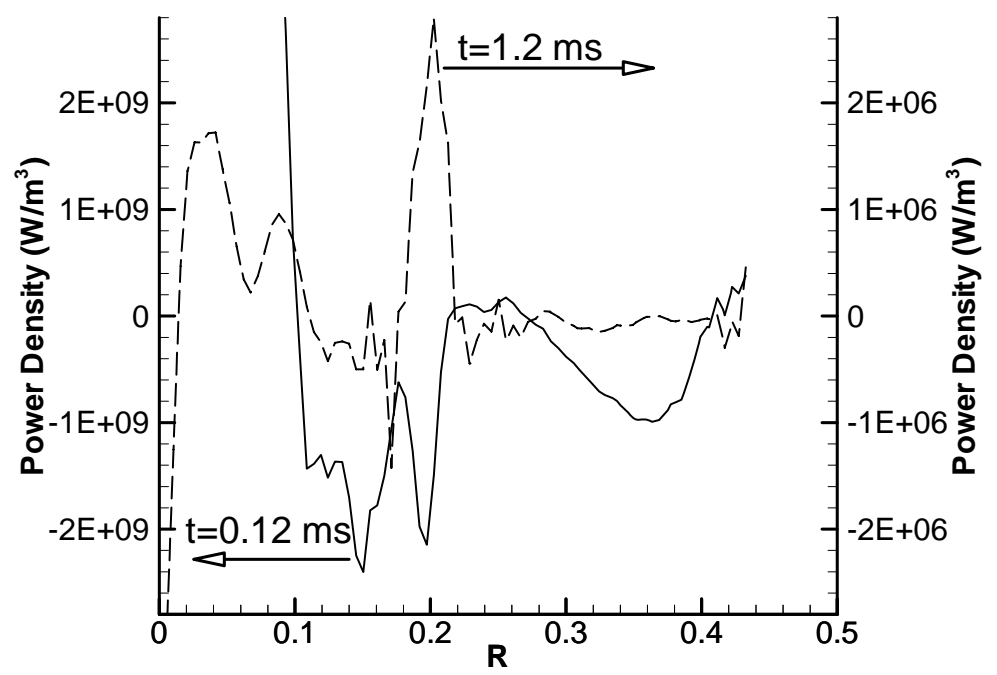

Figure 4. Dynamo power density, $-\langle\tilde{\mathbf{v}} \times \tilde{\mathbf{b}}\rangle \cdot\langle\mathbf{J}\rangle$, from the midplane of the NIMROD SSPX simulations during formation ( $t=0.12 \mathrm{~ms})$ and during the sustainment pulse $(t=1.2 \mathrm{~ms})$. The magnetic axis of the equilibrium is near $R=0.35 \mathrm{~m}$, and the fluctuations tend to drive current where the dynamo power density is negative. 


\subsection{Spherical torus current injection and start-up}

The start-up of small aspect ratio tokamaks, or spherical tori (ST), is an example where a transient excitation of MHD modes may be beneficial. With the configuration's small center stack, geometry and material properties limit the available magnetic flux swing for inductive current drive. This has led to exploration of electrostatic helicity injection for start-up in HIT-II at the University of Washington, in NSTX at Princeton Plasma Physics Laboratory, and in the Pegasus ST at the University of Wisconsinsee Refs. [26-28]. The start-up takes place in a manner similar to electrostatic drive for spheromaks, but the intent is to create an equilibrium from which Ohmic current drive can proceed efficiently. Depending on how the electrostatic injection is applied [26], MHD activity and magnetic relaxation [29,30] may be part of the process.

Modeling electrostatically assisted start-up in ST requires the ability to reproduce helicity injection in a vacuum toroidal field and Ohmic current drive, and significant progress has been made in this area. The former is similar to the current drive modeling in spheromaks described above and has been accomplished for cylindrical [31] and ST configurations [32]. To model the recent Pegasus experiments that have current injected from a miniature plasma gun [28], we have used the combined nonlinear MHD/collisional transport model with temperature-dependent resistivity. The gun is represented by localized electrostatic potential and temperature distributions on the bottom boundary of the domain (see Fig. 5), and vacuum toroidal and vertical magnetic fields are imposed. As the solution is advanced in time, the induced current flows along a helical channel with a pitch that is dictated by the vacuum field. Plasma along the helical channel is heated by Ohmic dissipation, so the temperature and conductivity distributions also become helically concentrated. Additional work is needed to model the merging of the helical channel as current is increased [28], where the self-induced fields compete with the applied vertical field.
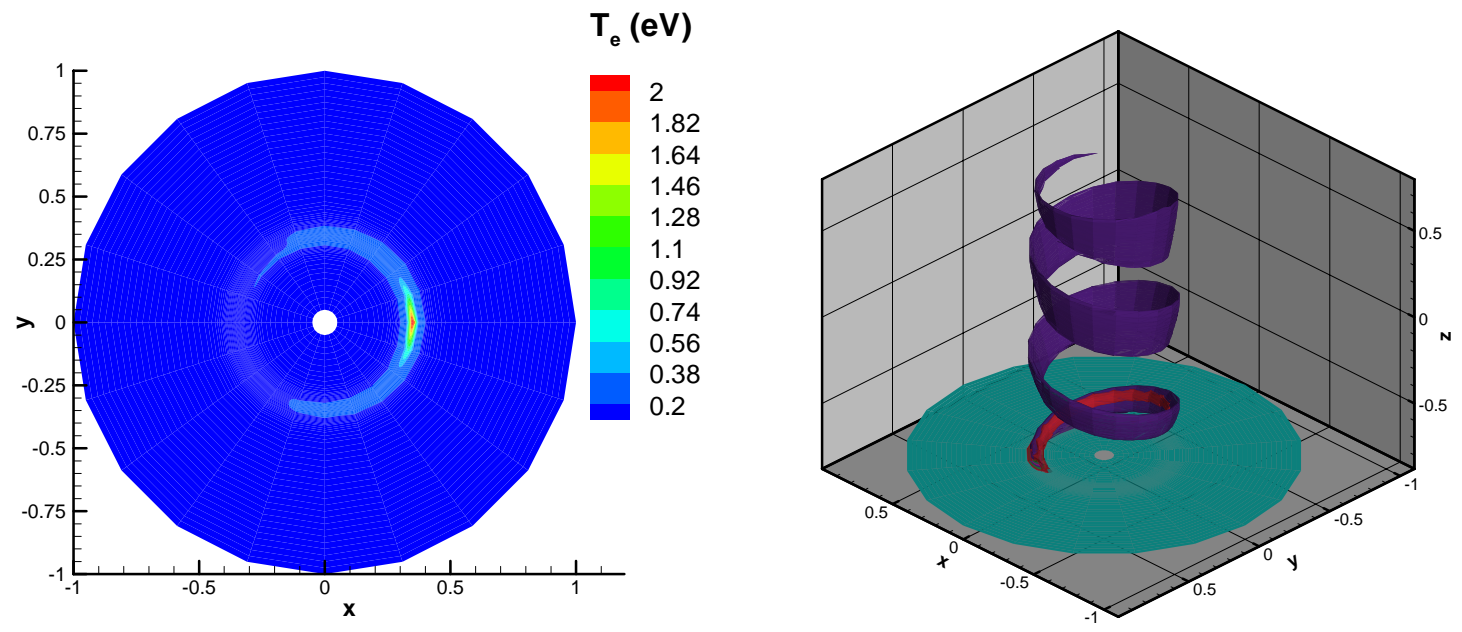

Figure 5. Contour plots of the applied temperature boundary condition (left) with a hot spot representing the miniature plasma gun in Pegasus. Isosurfaces of parallel current density ( $J_{\|} / B$, right) show the helical current channel that results with applied potential. 
The second requirement for modeling electrostatically assisted start-up is a realistic Ohmic-drive evolution. While it is easy to apply loop voltage in simulations with constant resistivity, the current distribution in tokamaks is heavily influenced by temperature-dependent effects. Simulations of Ohmic start-up have been completed with NIMROD including Spitzer resistivity and a realistic vertical-field evolution [33]. Anisotropic thermal conduction keeps the resistivity high in the open-field region, and current becomes localized to the growing region of nested closed-flux surfaces in a physically meaningful way.

\subsection{Numerical Modeling}

The simulations performed for this study have been carried-out with the NIMROD code (http://nimrodteam.org), which has been developed by a multi-institutional team through the support of other grants. A description and analysis of the numerical algorithm are provided in Ref. [24]. The code solves fluid-based models of plasma dynamics, and here we have used non-ideal magnetohydrodynamics:

$$
\begin{aligned}
& \frac{\partial n}{\partial t}+\nabla \cdot(n \mathbf{V})=\nabla \cdot D \nabla n \\
& \rho\left(\frac{\partial \mathbf{V}}{\partial t}+\mathbf{V} \cdot \nabla \mathbf{V}\right)=\mathbf{J} \times \mathbf{B}-\nabla p+\nabla \cdot \rho \nu \nabla \mathbf{V} \\
& \frac{n k}{\gamma-1}\left(\frac{\partial T}{\partial t}+\mathbf{V} \cdot \nabla T\right)=-\frac{p}{2} \nabla \cdot \mathbf{V}+\nabla \cdot n k\left[\chi \|_{\|} \hat{\mathbf{b}}+\chi_{\perp}(\mathbf{I}-\hat{\mathbf{b}} \hat{\mathbf{b}})\right] \cdot \nabla T+\frac{\eta \mathbf{J}^{2}}{2} \\
& \frac{\partial \mathbf{B}}{\partial t}=\nabla \times(\mathbf{V} \times \mathbf{B}-\eta \mathbf{J}) \\
& \mu_{0} \mathbf{J}=\nabla \times \mathbf{B}
\end{aligned}
$$

where $p \equiv 2 n k T$ is the sum of electron and ion pressures, and $\hat{\mathbf{b}} \equiv \mathbf{B} /|\mathbf{B}|$ is the evolving magnetic direction vector field. The simulations consider $n, T, \mathbf{V}, \mathbf{B}$, and $\mathbf{J}$ (number density, temperature, flow velocity, magnetic induction, and charge-current density, respectively) to be functions of all three spatial dimensions and time, so magnetic fluctuations and anisotropic heat flow (where used) are modeled explicitly. In the SSPX and Pegasus electrostatic current-injection simulations, the parallel and perpendicular thermal diffusivities are temperature dependent, $\chi_{\|}=387 T^{5 / 2} \mathrm{~m}^{2} / \mathrm{s}$ and $\chi_{\perp}=0.50 T^{-1 / 2} B^{-2} \mathrm{~m}^{2} / \mathrm{s}$ ( $B$ in Tesla) based on electrons and ions [23], respectively, for a hydrogen plasma at $n=5 \times 10^{19} \mathrm{~m}^{-3}$. The numerical computation of $\chi_{\perp}$ is simplified by using the toroidal average (indicated by \langle\rangle , hereafter) of the evolving temperature and magnetic induction fields. The numerical capability of modeling temperature-dependent collisional heat-transport has been developed through support from this grant. The code modifications have been made available to collaborators and to the NIMROD Team.

Some of the PPCD simulations have also been conducted with finite plasma pressure; however, most have focused on current-driven MHD activity, and a simplified model is used. Equations (2), (4), and (5) are evolved without the pressure gradient term in (2), which represents the low- $\beta$, high compressibility limit of the MHD model. 


\subsection{References}

1. B. E. Chapman, A. F. Almagri, J. K. Anderson, T. M. Biewer, P. K. Chattopadhyay, C.-S. Chiang, D. J. Craig, D. J. Den Hartog, G. Fiksel, C. B. Forest, A. K. Hanson, D. Holley, N. Lanier, R. O’ Connell, S. C. Prager, J. C. Reardon, J. S. Sarff, M. D. Wyman, D. L. Brower, W. X. Ding, Y. Jiang, S. D. Terry, P. Franz, L. Marrelli, and P. Martin, "High confinement plasmas in the Madison Symmetric Torus reversed-field pinch,” Phys. Plasmas 9, 2061 (2002).

2. J. S. Sarff, S. A. Hokin, H. Ji, S. C. Prager, and C. R. Sovinec, "Fluctuation and transport reduction in a reversed field pinch by inductive poloidal current drive,” Phys. Rev. Lett. 72, 3670 (1994).

3. J. K. Anderson, T. M Biewer, C. B. Forest, R. O’Connell, S. C. Prager, and J. S. Sarff, "Dynamo-free plasma in the reversed field pinch,” Phys. Plasmas 11, L9 (2004).

4. J. Reynolds and C. Sovinec, "Computational studies of MHD dynamo suppression in the reversed field pinch during PPCD,” Bull. Am. Phys. Soc. 49, 8, p. 272 (2004).

5. M. R. Stoneking, N. E. Lanier, S. C. Prager, J. S. Sarff, and D. Sinitsyn, "Fivefold confinement time increase in the Madison Symmetric Torus using inductive poloidal current drive,” Phys. Plasmas 4, 1632 (1997).

6. J. A. Holmes, B. A. Carreras, P. H. Diamond, and V. E. Lynch, "Nonlinear dynamics of tearing modes in the reversed field pinch,” Phys. Fluids 31, 1166 (1988).

7. Y. L. Ho and G.G. Craddock, "Nonlinear dynamics of field maintenance and quasiperiodic relaxation in reversed-field pinches,” Phys. Fluids B 3, 721 (1991).

8. H. P. Furth, J. Killeen, and M. N. Rosenbluth, "Finite-resistivity instabilities of a sheet pinch,” Phys. Rev. Lett. 6, 459 (1963).

9. D. C. Robinson, “Tearing-mode stable diffuse-pinch configurations,” Nucl. Fusion 18, 939 (1978).

10. M. E. Puiatti, S. Cappello et al. "Analysis and modeling of the magnetic and plasma profiles during PPCD experiments in RFX,” Nuclear Fusion 43, 1057 (2003).

11. S. O. Knox, C. W. Barnes, G. J. Marklin, T. R. Jarboe, I. Henins H. W. Hoida, and B. L. Wright, "Observations of spheromak equilibria which differ from the minimum energy state and have internal kink distortions,” Phys. Rev. Lett. 56, 842 (1986).

12. M. Nagata, T. Kanki, T. Masuda, S. Naito, H. Tatsumi, and T. Uyama, "Relaxation oscillations and toroidal-current regeneration in a helicity-driven spheromak,” Phys. Rev. Lett. 71, 4342 (1993).

13. R. C. Duck, P. K. Browning, G. Cunningham, S. J. Gee, A. al-Karkhy, R. Martin, and M. G. Rusbridge, "Structure of the $\mathrm{n}=1$ mode responsible for relaxation and current drive during sustainment of the SPHEX spheromak," Plasma Phys. Control. Fusion 39, 715 (1997).

14 T. R. Jarboe, F. J. Wysocki, J. C. Fernandez, I. Henins, and G. J. Marklin, "Progress with energy confinement time in the CTX spheromak,” Phys. Fluids B 2, 1342 (1990).

15. H. S. McLean, S. Woodruff, E. B. Hooper, R. H. Bulmer, D. N. Hill, C. Holcomb, J. Moller, B. W. Stallard, R. D. Wood, and Z. Wang, "Suppression of MHD fluctuations leading to improved confinement in a gun-driven spheromak,” Phys. Rev. Lett. 88, 125004 (2002).

16. H. S. McLean et al., in Proceedings of the $30^{\text {th }}$ EPS Conference on Contr. Fusion and Plasma Phys., St. Petersburg, Russia, ECA Vol. 27A, 3.230 (2003); S. Woodruff, private communication.

17. E. B. Hooper, L. D. Pearlstein, and R. H. Bulmer, "MHD equilibria in a spheromak sustained by coaxial helicity injection,” Nucl. Fusion 39, 863 (1999). 
18. C. R. Sovinec, J. M. Finn, and D. Del-Castillo-Negrete, "Formation and sustainment of electrostatically driven spheromaks in the resistive magnetohydrodynamic model,” Phys. Plasmas 8 , 475 (2001).

19. J. M. Finn, C. R. Sovinec, and D. Del-Castillo-Negrete, "Chaotic scattering and self-organization in spheromak sustainment,” Phys. Rev. Lett. 85, 4538 (2000).

20. E. B. Hooper, R. H. Cohen, and D. D. Ryutov, “Theory of edge plasma in a spheromak,”J. Nucl. Mat. 278, 104 (2000).

21. R. W. Moses, R. A. Gerwin, and K. F. Schoenberg, "Transport implications of current drive by magnetic helicity injection,” Phys. Plasmas 8, 4839 (2001).

22. C. R. Sovinec, B. I. Cohen, G. A. Cone, E. B. Hooper, and H. S. McLean, "Numerical Investigation of Transients in the SSPX Spheromak,” Physical Review Letters 94, 35003 (2005).

23. S. I. Braginskii, “Transport Processes in a Plasma,” Reviews of Plasma Physics, 1, 205 (Consultants Bureau, New York, 1965).

24. C. R. Sovinec, A. H. Glasser, T. A. Gianakon, D. C. Barnes, R. A. Nebel, S. E. Kruger, D. D. Schnack, S. J. Plimpton, A. Tarditi, M. Chu, and the NIMROD Team, "Nonlinear Magnetohydrodynamics Simulation using High-Order Finite Elements,” J. Comput. Phys. 195, 355 (2004).

25. B. I. Cohen, E. B. Hooper, R. H. Cohen, D. N. Hill, H. S. McLean, R. D. Wood, S. Woodruff, C. R. Sovinec, and G. A. Cone, "Simulation of Spheromak Evolution and Energy Confinement," accepted for publication in Physics of Plasmas.

26. R. Raman, T. R. Jarboe, B. A. Nelson, W. T. Hamp, V. A. Izzo, R. G. O’Neill, A. J. Redd, P. E. Sieck, and R. J. Smith, "Experimental demonstration of plasma startup by coaxial helicity injection,” Phys. Plasmas 11, 2565 (2004).

27. R. Raman, T. R. Jarboe, B. A. Nelson, M. Bell, D. Mueller, and M. Schaffer, "Solenoid free plasmas start-up in NSTX by coaxial helicity injection,” Bull. Am. Phys. Soc. 49, 8, p. 68 (2004).

28. N. W. Eidietis, S. P. Burke, G. Fiksel, R. J. Fonck, G. D. Garstka, E. A. Unterberg, and G. R. Winz, "Fueling and plasma initiation test with a plasma gun on the PEGASUS toroidal experiment," Bull. Am. Phys. Soc. 49, 8, p. 304 (2004).

29. B. A. Nelson, T. R. Jarboe, D. J. Orvis, L. A. McCullough, J. Xie, C. Zhang, and L. Zhou, "Formation and sustainment of a 150 kA tokamak by coaxial helicity injection,” Phys. Rev. Lett. 72, 3666 (1994).

30. T. R. Jarboe, M. A. Bohnet, A. T. Mattick, B. A. Nelson, and D. J. Orvis, "Results from current drive experiments on the Helicity Injected Torus,” Phys. Plasmas 5, 1807 (1998).

31. C. R. Sovinec and S. C. Prager, "Magnetohydrodynamic simulations of direct current helicity injection for current drive in tokamaks,” Phys. Plasmas 3, 1038 (1996).

32. X. Tang and A. H. Boozer, "Current drive by coaxial helicity injection in a spherical torus," Phys. Plasmas 11, 2679 (2004).

33. C. R. Sovinec, T. A. Gianakon, E. D. Held, S. E. Kruger, D. D. Schnack, and the NIMROD Team, "NIMROD: a Computational Laboratory for Studying Nonlinear Fusion Magnetohydrodynamics," Phys. Plasmas 10, 1727(2003). 


\section{Products}

include:

Refereed journal publications resulting from this study and from closely related collaborative efforts

- C. R. Sovinec, T. A. Gianakon, E. D. Held, S. E. Kruger, D. D. Schnack, and the NIMROD Team, "NIMROD: a Computational Laboratory for Studying Nonlinear Fusion Magnetohydrodynamics,” Phys. Plasmas 10, 1727 (2003).

- C. R. Sovinec, B. I. Cohen, G. A. Cone, E. B. Hooper, and H. S. McLean, "Numerical Investigation of Transients in the SSPX Spheromak," Physical Review Letters 94, 35003 (2005).

- S. Woodruff, B. I. Cohen, E. B. Hooper, H. S. McLean, B. W. Stallard, D. N. Hill, C. T. Holcomb, C. Romero-Talamaras, R. D. Wood, G. Cone, and C. R. Sovinec, "Controlled and Spontaneous Magnetic Field Generation in a Gun-Driven Spheromak,” Physics of Plasmas 12, 52505 (2005).

- B. I. Cohen, E. B. Hooper, R. H. Cohen, D. N. Hill, H. S. McLean, R. D. Wood, S. Woodruff, C. R. Sovinec, and G. A. Cone, "Simulation of Spheromak Evolution and Energy Confinement," Physics of Plasmas 12, 56106 (2005).

- $\quad$ E. B. Hooper, T. A. Kopriva, B. I. Cohen, D. N. Hill, H. S. McLean, R. D. Wood, S. Woodruff, and C. R. Sovinec, "Magnetic Reconnection during Flux Conversion in a Driven Spheromak," accepted for publication in Physics of Plasmas.

Invited conference presentations on this work and closely related collaborations include:

- C. R. Sovinec, “Spheromak Transients and Energy Confinement,” Innovative Confinement Concepts Workshop, Madison, WI, May 25, 2004.

- C. R. Sovinec, "Spheromak Energy Confinement in Sustained and Transient Conditions," $31^{\text {st }}$ IEEE International Conference on Plasma Science, Baltimore, MD, June 28, 2004.

- B. I. Cohen, "Simulation of Spheromak Evolution and Energy Confinement," $46^{\text {th }}$ Annual Meeting of the APS Division of Plasma Physics, Savannah, GA, November 18, 2004.

- C. R. Sovinec, "Comparing Transient High-Confinement States in Spheromak and RFP Discharges," $9^{\text {th }}$ Easter Plasma Meeting on Stability and Confinement of Magnetized Plasmas, Turin, Italy, April 1, 2005.

Relevant presentations are available from http://www.cptc.wisc.edu/Sovinec_research and from http://nimrodteam.org.

This computational effort has fostered a productive collaboration between the Center for Plasma Theory and Computation (CPTC) at the University of Wisconsin-Madison and the magnetic fusion energy group at Lawrence Livermore National Laboratory (LLNL). The spheromak modeling techniques developed at CPTC have been transferred to researchers at LLNL, and LLNL personnel have provided laboratory data on SSPX, in addition to carrying-out complementary spheromak simulation studies. An undergraduate student from Wisconsin, Thomas Kopriva, also spent a summer working on spheromak simulation analysis with the LLNL group. 